\title{
One step too far in the right direction: The Society for Translational Medicine 2019 consensus on postoperative management of EGFR-mutant lung cancer
}

\author{
Daniel Breadner ${ }^{1,2}$, Jacques Raphael ${ }^{1,2}$ \\ ${ }^{1}$ Division of Medical Oncology, London Regional Cancer Program at London Health Science Center, London, Canada; ${ }^{2}$ Schulich School of \\ Medicine and Dentistry at Western University, London, Canada \\ Correspondence to: Daniel Breadner. A3-941, LRCP, Medical Oncology, 800 Commissioners Road East, London, Ontario, N6A5W9, Canada. \\ Email: Daniel.breadner@1hsc.on.ca. \\ Provenance and Peer Review: This article was commissioned by the editorial office, fournal of Thoracic Disease. The article did not undergo external peer review. \\ Comment on: Liang W, Cai K, Chen C, et al. Society for Translational Medicine consensus on postoperative management of EGFR-mutant lung \\ cancer (2019 edition). Transl Lung Cancer Res 2019;8:1163-73.
}

Submitted Apr 27, 2020. Accepted for publication May 09, 2020.

doi: 10.21037/jtd-2020-51

View this article at: http://dx.doi.org/10.21037/jtd-2020-51

Resected non-small cell lung cancer (NSCLC) remains a serious cause of morbidity and mortality with about half of resected patients developing recurrent disease during their lifetime (1). Patients with NSCLC whose tumors harbor activating EGFR mutations (EGFR+) are managed much differently in the metastatic setting than EGFR wildtype NSCLC, utilizing upfront treatment with EGFR tyrosine kinase inhibitors (TKIs). EGFR-TKIs provide a significant improvement in progression free survival (PFS) compared to chemotherapy with greater than a $50 \%$ reduction in the risk for progression in the treatment of EGFR+ metastatic NSCLC (2). Osimertinib, a third-generation EGFR-TKI, has superior efficacy and tolerability compared to earlier generation EGFR-TKIs and is now the first-line standard of care (3). Considering the improved efficacy and tolerance of EGFR-TKIs compared to cytotoxic chemotherapy, in the metastatic setting, it is a natural step to explore the use of EGFR-TKIs in the adjuvant setting.

The Society for Translational Medicine's (The Society's) 2019 consensus on postoperative management of EGFRmutant lung cancer endorses the testing of resected NSCLC for EGFR mutations, EGFR-TKIs as a treatment option and suggestions on the surveillance strategy for patients with resected EGFR+ NSCLC and some management recommendations for patients with recurrent disease (4). These recommendations cover a broad aspect of care, especially when dealing with resected EGFR+ NSCLC where the strength of evidence varies. This is highlighted by the accompanying commentary of non-author experts, many of whom descent from or add caveats to The Society's recommendations.

Consensus statements \#3 and 4 provide a strong recommendation for adjuvant EGFR-TKIs as a treatment option for patients with resected stage Ib-IIIA EGFR+ NSCLC, either in place of or following cytotoxic chemotherapy. A recent meta-analysis of six eligible randomized control trials reported a consistent disease-free survival (DFS) benefit, but no overall survival (OS) benefit (5). It is important to consider that there is an absence of reliable OS benefit from any of the randomized trials (6-9). The EVAN trial, testing erlotinib versus cisplatin and vinorelbine in 102 patients with stage III disease, is the only trial to report a survival benefit, although that data is still immature with only three and 13 deaths in the two arms, respectively, and 18 of 51 chemotherapy patients' treatment not adhering to protocol (8). It is our opinion that the intent of an adjuvant treatment is to increase the chance of cure. Awaiting the final OS analysis from the landmark adjuvant EGFR-TKI trials is crucial to assess their impact on treatment. If treatment does not improve OS then it is giving toxicity to all patients and delaying the recurrence of some without prolonging life. Therefore, in the absence of improving OS the only rationale for that adjuvant treatment would be robust positive quality of life data and patient 
preference.

Another difficulty with the use of adjuvant EGFR-TKIs is the timing and duration of treatment. Some studies have compared EGFR-TKIs to placebo with some patients receiving adjuvant chemotherapy, while other studies have compared EGFR-TKIs to chemotherapy. A well designed and pragmatic trial would randomize patients to either chemotherapy or an EGFR-TKI or chemotherapy followed by an EGFR-TKI. If this trial supported the superiority of EGFR-TKIs then determining the optimal duration in a clinical trial setting would be the best next step. The consensus statement \#5 recommends at least two years of adjuvant therapy, as most trials gave two years of EGFR-TKI therapy and the recurrences seem to occur at a higher rate once therapy was stopped. A phase II open label study randomizing patients to 6 months of gefitinib or direct to observation after carboplatin and pemetrexed reported a strong DFS benefit with a trend towards OS improvement and the benefit appeared to be sustained well beyond 2 years (7). In the RADIANT trial the median treatment duration was less than a year (6), mostly because of toxicity, highlighting that EGFRTKIs are not benign and the optimal duration of therapy remains unknown and needs to be properly studied.

A difficulty in making strong recommendations from the existing body of literature is the heterogeneity within research. Some studies test EGFR-TKIs versus placebo in a mixed population of those that did or did not receive adjuvant chemotherapy, where other studies tested adjuvant EGFR-TKIs versus chemotherapy. The existing trials have largely been done in a limited geographic area and do not contain a strong balance of Asian and non-Asian patients. The ADAURA study will add intriguing knowledge to this body of research (10). It includes a balance of patients from Asia, North America, Europe and Australia and tests osimertinib versus placebo after any planned adjuvant chemotherapy. ADAURA results are expected soon, as its monitoring committee has recommended unblinding and early reporting. Osimertinib is a better tolerated EGFRTKI, and is superior in the metastatic setting, than firstgeneration EGFR-TKIs (3) but ADAURA cannot answer whether EGFR-TKIs can replace adjuvant chemotherapy.

The Society recommends the addition of annual MRI brain and bone scan to routine surveillance. We agree that patients with EGFR+ NSCLC are at increased risk of recurrent disease in the bones or brain, but surveillance guidelines should be guided by the principle that the intervention, and the accompanying costs and anxiety for patients, yield improved outcomes. We appreciate that stereotactic radiation to oligometastatic or limited recurrent disease can be treated to improve survival (11), and supports a well-designed trial of different surveillance strategies. In the absence of evidence supporting more aggressive surveillance, it is reasonable to ask clinicians and patients to consider adding MRI brain imaging and bone scans to routine surveillance, but we do not feel it warrants a strong recommendation.

Consensus statements \#7 and 8 make recommendations regarding the utility of liquid biopsy and the use of osimertinib in recurrent disease or retreatment with the adjuvant TKI if the disease recurrence occurs after adjuvant therapy discontinuation. We agree that circulating tumor DNA (ctDNA) analysis may be an appropriate tool to confirm the recurrent disease is EGFR+, especially in a patient at risk for a second malignancy, as ctDNA testing may replace the need for an invasive biopsy (12). Recommending retreatment in the advanced setting with the same EGFR-TKI used in the adjuvant setting may be premature. It is encouraging that the SELECT trial reported a high success rate of retreatment with erlotinib (13), and this strategy is supported by scientific rationale and EGFR-TKI rechallenge in the metastatic setting which is undergoing more rigorous investigation (14-16).

Furthermore, the implications on metastatic management after treatment with EGFR-TKIs in the adjuvant setting needs to be investigated. If the use of EGFR-TKIs in the metastatic setting yields a markedly shorter PFS than in EGFR-TKI naïve patients it would explain the lack of OS in most adjuvant EGFR-TKI studies. It is not known how the EGFR-TKI used in the adjuvant setting and the duration off treatment, prior to the discovery of recurrent disease, will affect the efficacy of EGFR-TKIs in the metastatic setting and when clinicians should offer rebiopsy to rule out the presence of a T790M mutation. The FLAURA trial has certainly established osimertinib as the optimal treatment in EGFR+ mNSCLC in patients that are EGFR-TKI naïve (3), but there is not high level evidence for the optimal treatment after failure of adjuvant EGFRTKIs, although osimertinib is certainly an appealing option in the absence of any evidence to the contrary.

In the future it is possible that ctDNA analysis may affect patients with resected EGFR+ NSCLC, allowing for the post-operative evaluation of molecular residual disease (MRD) (17). The validity of existing adjuvant EGFR-TKI trials would need to be re-examined if the adjuvant standard of care evolves to include the assessment of MRD.

Overall the consensus statements do an excellent job at highlighting that the evidence for adjuvant EGFR- 
TKIs is building, and with the addition of ADAURA and maturing survival data from previously reported trials the management of adjuvant EGFR+ NSCLC is likely to soon be changing. However, the evidence at this point has not established if EGFR-TKIs can replace chemotherapy and the absence of convincing and widely applicable OS benefit it would be hard to expect widespread adoption of adjuvant EGFR-TKIs. Although we expect that the management of resected EGFR+ NSCLC will change in the future, we do not feel that the existing body of knowledge warrants expansive use of adjuvant EGFR-TKIs at this time.

\section{Acknowledgments}

Funding: None.

\section{Footnote}

Conflicts of Interest: Both authors have completed the ICMJE uniform disclosure form (available at http://dx.doi. org/10.21037/jtd-2020-51). JR reports personal fees from Advisory board, personal fees from Honorarium, outside the submitted work. The other author has no conflicts of interest to declare.

Ethical Statement: The authors are accountable for all aspects of the work in ensuring that questions related to the accuracy or integrity of any part of the work are appropriately investigated and resolved.

Open Access Statement: This is an Open Access article distributed in accordance with the Creative Commons Attribution-NonCommercial-NoDerivs 4.0 International License (CC BY-NC-ND 4.0), which permits the noncommercial replication and distribution of the article with the strict proviso that no changes or edits are made and the original work is properly cited (including links to both the formal publication through the relevant DOI and the license). See: https://creativecommons.org/licenses/by-nc-nd/4.0/.

\section{References}

1. Pignon JP, Tribodet H, Scagliotti GV, et al. Lung adjuvant cisplatin evaluation: a pooled analysis by the LACE Collaborative Group. Database of Abstracts of Reviews of Effects (DARE): Quality-assessed Reviews. Centre for Reviews and Dissemination (UK), 2008.

2. Haspinger ER, Agustoni F, Torri V, et al. Is there evidence for different effects among EGFR-TKIs? Systematic review and meta-analysis of EGFR tyrosine kinase inhibitors (TKIs) versus chemotherapy as first-line treatment for patients harboring EGFR mutations. Crit Rev Oncol Hematol 2015;94:213-27.

3. Ramalingam SS, Yang J, Lee CK, et al. Osimertinib as first-line treatment of EGFR mutation-positive advanced non-small-cell lung cancer. J Clin Oncol 2018;36:841-9.

4. Liang W, Cai K, Chen C, et al. Society for Translational Medicine consensus on postoperative management of EGFR-mutant lung cancer (2019 edition). Transl Lung Cancer Res 2019;8:1163-73.

5. Raphael J, Vincent M, Boldt G, et al. Adjuvant Epidermal Growth Factor Receptor Tyrosine Kinase Inhibitors (TKIs) in Resected Non-Small Cell Lung Cancer (NSCLC). Am J Clin Oncol 2019;42:440-5.

6. Kelly K, Altorki NK, Eberhardt WE, et al. Adjuvant erlotinib versus placebo in patients with stage IB-IIIA nonsmall-cell lung cancer (RADIANT): A randomized, double-blind, Phase III trial. J Clin Oncol 2015;33:400714.

7. Li N, Ou W, Ye X, et al. Pemetrexed-carboplatin adjuvant chemotherapy with or without gefitinib in resected stage IIIA-N2 non-small cell lung cancer harbouring EGFR mutations: a randomized, phase II study. Ann Surg Oncol 2014;21:2091-6.

8. Yue D, Xu S, Wang Q, et al. Erlotinib versus vinorelbine plus cisplatin as adjuvant therapy in Chinese patients with stage IIIA EGFR mutation-positive non-small-cell lung cancer (EVAN): a randomised, open-label, phase 2 trial. Lancet Respir Med 2018;6:863-73.

9. Zhong WZ, Wang Q, Mao WM, et al. Gefitinib versus vinorelbine plus cisplatin as adjuvant treatment for stage II-IIIA (N1-N2) EGFR-mutant NSCLC (ADJUVANT/ CTONG1104): a randomised, open-label, phase 3 study. Lancet Oncol 2018;19:139-48.

10. AZD9291 Versus Placebo in Patients With Stage IBIIIA Non-small Cell Lung Carcinoma, Following Complete Tumour Resection With or Without Adjuvant Chemotherapy. Available online: https://ClinicalTrials. gov/show/NCT02511106.

11. Palma DA, Olson R, Harrow S, et al. Stereotactic ablative radiotherapy versus standard of care palliative treatment in patients with oligometastatic cancers (SABRCOMET): a randomised, phase 2, open-label trial. Lancet 2019;393:2051-8.

12. Leighl NB, Page RD, Raymond VM, et al. Clinical Utility of Comprehensive Cell-free DNA Analysis to Identify 
Genomic Biomarkers in Patients with Newly Diagnosed Metastatic Non-small Cell Lung Cancer. Clin Cancer Res 2019;25:4691-700.

13. Pennell NA, Neal JW, Chaft JE, et al. SELECT: a phase II trial of adjuvant erlotinib in patients with resected epidermal growth factor receptor-mutant non-small-cell lung cancer. J Clin Oncol 2019;37:97-104.

14. Osimertinib Then Chemotherapy in EGFR-mutated Lung Cancer With Osimertinib Third-line Rechallenge. Available online: https://ClinicalTrials.gov/show/ NCT04335292.

15. Kuiper JL, Heideman DA, Würdinger T, et al. Rationale and study design of the IRENE-trial (NVALT-16): a phase
II trial to evaluate iressa rechallenge in advanced NSCLC patients with an activating EGFR mutation who responded to an EGFR-TKI used as first-line or previous treatment. Clin Lung Cancer 2015;16:60-6.

16. Oda N, Ichihara E, Hotta K, et al. Phase II Study of the EGFR-TKI Rechallenge With Afatinib in Patients With Advanced NSCLC Harboring Sensitive EGFR Mutation Without T790M: Okayama Lung Cancer Study Group Trial OLCSG 1403. Clin Lung Cancer 2017;18:241-4.

17. Chaudhuri AA, Chabon JJ, Lovejoy AF, et al. Early Detection of Molecular Residual Disease in Localized Lung Cancer by Circulating Tumor DNA Profiling. Cancer Discov 2017;7:1394-403.
Cite this article as: Breadner D, Raphael J. One step too far in the right direction: The Society for Translational Medicine 2019 consensus on postoperative management of EGFR-mutant lung cancer. J Thorac Dis 2020;12(9):5046-5049. doi: 10.21037/jtd2020-51 